\title{
Muséologies
}

Les cahiers d'études supérieures

\section{Colloque étudiant en muséologie de l'UQAM et de l'UDEM : Le rôle social du musée}

\section{Émilie Allain, Eve Lyne Cayouette Ashby, Nellie Demers et Virginie Soulier}

Volume 2, numéro 2, avril 2008

URI : https://id.erudit.org/iderudit/1033592ar

DOI : https://doi.org/10.7202/1033592ar

Aller au sommaire du numéro

Éditeur(s)

Association Québécoise de Promotion des Recherches Étudiantes en

Muséologie (AQPREM)

ISSN

1718-5181 (imprimé)

1929-7815 (numérique)

Découvrir la revue

Citer ce compte rendu

Allain, É., Cayouette Ashby, E. L., Demers, N. \& Soulier, V. (2008). Compte rendu de [Colloque étudiant en muséologie de l'UQAM et de l'UDEM : Le rôle social du musée]. Muséologies, 2(2), 112-117. https://doi.org/10.7202/1033592ar 
Billet un

\section{Colloque étudiant en muséologie de I'UQAM et de I'UDEM : Le rôle social du musée}

[Par Émilie Allain, Eve Lyne Cayouette Ashby, Nellie Demers, Virginie Soulier] 
Depuis les années 1970, un vaste mouvement de muséologie sociale a émergé de certaines institutions muséales et les a orientées de différentes manières : démocratisation et accès, collaboration avec les communautés. Le musée, " temple du beau ", devait céder la place au " musée forum ", lieu de débats et de rencontres ${ }^{[1]}$. Qu'en est-il aujourd'hui, presque 40 ans plus tard ? Le musée est-il démocratique et adapté à tous ? Dans nos sociétés cosmopolites et diversifiées, à qui le musée s'adresse-t-il ? Quel genre de relations entretient-il avec la communauté ? Bref, quel est son rôle, sa place dans l'espace public ${ }^{[2]}$ ?

\begin{abstract}
À la suite de la journée de réflexion sur cette thématique, les organisatrices du colloque ont demandé aux présidents de séance et aux conférenciers de présenter leur point de vue rétroactif. Nous vous proposons ainsi un bilan concis et critique des idées développées, reflétant les rôles et les démarches actuelles de certains musées du Québec vis-à-vis de leur société en mutation et ce, de points de vue tant local que global.
\end{abstract}

\section{[1]}

CAMERON, Duncan.

"Le musée : temple ou forum". In. DESVALLÉES, André (dir.).

Vagues 1. Une anthologie

de la nouvelle muséologie.

Mâcon : Éditions W/MNES, 1992.

\section{[2]}

Appel de propositions au colloque. 


\section{De la nouvelle muséologie à la}

\section{muséologie participative}

Les années $1970-1980^{[3]}$ ont contribué à la prise de conscience de l'importance des questions concernant le public - comme le rôle socio-éducatif du musée, l'accessibilité aux collections et à la recherche, les responsabilités des musées vis-à-vis de l'actualité, des échanges et des débats publics et à l'élaboration d'un système de communication et de médiation ${ }^{[4]}$. Cette période a introduit les laissés-pour-compte dans l'enceinte du musée, les "kystes culturels" " ${ }^{[5]}$ ou les "Autres", commeles qualifie Hugues de Varine. La nouvelle muséologie a ainsi défini des orientations aux antipodes de ce qu'était jusqu'alors un musée " temple". L'idéal du musée "forum " propose une vision globale de l'homme tournée vers le présent et l'avenir. Les notions clés l'éclairant et ses impacts actuels sont la popularisation, l'absence de collection, la virtualité, l'oralité, l'accessibilité, la pluridisciplinarité.
Le développement industriel et marchand du secteur des communications sociales et du marketing s'est allié aux préoccupations de la démocratisation culturelle. Le rôle social du musée se situe aujourd'hui dans cette volonté d'améliorer l'offre, pour attirer et retenir les visiteurs, et de s'assurer que cette offre est " arrimée » à la société. Il faut pour cela connaître, comprendre et être en étroite relation avec la société pour la faire parler, l'écouter et apprendre d'elle. Mais on en arrive très vite au problème de définition de la société ellemême et des enjeux sociaux et culturels liés à la mondialisation et aux migrations. Le défi actuel des musées est d'établir un équilibre entre une population plurielle locale et un tourisme global, d'où la tendance au "glocal».

De nouvelles pratiques muséologiques sont nées des fondements de la nouvelle muséologie, telles l'écomuséologie, la muséologie sociale et la muséologie participative ${ }^{[6]}$.

\section{[3]}

La définition de l'ICOM (Conseil international des musées) est née de cette perspective.

\section{[4]}

CAMERON, Duncan.

"Un point de vue : le musée considéré comme système de communication et les implications de ce système dans les programmes éducatifs muséaux (1968)». In. DESVALLÉES, André (dir.). Vagues 1. Une anthologie de la nouvelle muséologie. Mâcon : Éditions W/MNES, 1992.
[5]

DE VARINE, Hugues. La culture des autres. Paris : Seuil, 1976, p.17.
[6]

Un mouvement s'est créé pour mettre en pratique l'esprit de la nouvelle muséologie : le Mouvement international pour une nouvelle muséologie, dit le MINOM, <http:// cadernosociomuseologia. ulusofona.pt/Arquivo/ caderno_22/cadernos_petit_ lexique_museologies.htm> (consulté en février 2008). 


\section{Les actions : dialogue, participation}

\section{et dématérialisation}

Lorsque les idéaux prennent forme, ils donnent naissance à une variété de projets qui permettent au musée de jouer activement son rôle social en contribuant à la socialisation et au développement de la citoyenneté sur son territoire. Les démarches de la muséologie participative étaient très présentes pendant le colloque, aspirant à rendre "l'Autre» dans le "Nous " et faisant partie du "tout le monde». La permanence sociale des musées se reflétant dans leurs expositions historiques ou d'art et leur utilisation politique par les gouvernements font de ces institutions des lieux d'apprentissage d'une histoire nationale et internationale officielle et même quelquefois superficielle. À l'instar de plusieurs autres secteurs de la recherche en sciences humaines et sociales ${ }^{[7]}$, il a été suggéré que la muséologie se penche plus vigoureusement sur la présence et l'histoire des communautés culturelles et de la diversité sociale du Québec. La majorité des initiatives présentées lors de cette journée concernait ainsi la participation des non-publics et des minorités, leur autonomisation et la naissance d'un dialogue et d'un partage d'autorité entre le musée et ses publics, grâce à des actions extra muros, à l'implication de non-professionnels, aux échanges intergénérationnels et interculturels et, quelquefois, à la mise en œuvre de situations d'apprentissages mutuels.

Ce dialogue permet d'ailleurs au musée de mieux connaître les besoins de ses publics et, surtout, de ses non-publics et d'y répondre de façon appropriée. Qu'il s'agisse de médiation de l'art contemporain ou d'intégration du public adolescent et du bénévolat, l'objectif est le même : accessibilité et démocratisation culturelle. La société change et le musée aussi. Le musée n'est ainsi plus un " temple " ou un « forum», mais, comme le mentionne Laurent Gervereau, il deviendrait

\section{[7]}

PROST, Antoine.

Douze leçons sur l'histoire. Paris : Seuil, 1996, p. 37-41 Les Annales et I'histoirerecherche, "La nouveauté des Annales n'est pas dans la méthode, mais dans les objets et les questions. L. Febvre et M. Bloch respectent parfaitement les normes de la profession; ils travaillent sur documents et citent leurs sources [...] Mais ils critiquent l'étroitesse des interrogations et le cloisonnement des investigations. Ils refusent l'histoire politique événementielle [...] pour mieux lui opposer une histoire largement ouverte, une histoire totale qui prenne en charge tous les aspects de l'activité humaine. Cette histoire 'économique et sociale', pour reprendre le titre de la nouvelle revue, se veut accueillante aux autres disciplines; à la sociologie, à l'économie; à la géographie. Histoire vivante, elle s'intéresse directement aux problèmes contemporains.» 
un "musée-reflet de vie» " ${ }^{[8]}$, surtout en contexte urbain; mutant à la mesure des attentes nouvelles des publics avec des offres polymorphes in situ et investissant son territoire tout en parlant au monde et en sortant de ses murs.

Différents outils méthodologiques, notamment l'inventaire participatif du patrimoine ${ }^{[0]}$, sont proposés. Ces outils permettraient d'atteindre une certaine autonomie de représentation par la participation des populations aux processus muséologique et muséographique et ce, tant localement que dans les grandes expositions internationales.

Par ailleurs, la "dématérialisation " du musée est également soulignée. L'omniprésence des nouvelles technologies et leurs impacts sur le monde muséal sont démontrés, comme la dépossession des propriétés intellectuelles des œuvres numériques, mais également l'importance croissante du patrimoine immatériel dans la valorisation et la construction de la mémoire des groupes minoritaires. Musées virtuels, œuvres d'art numériques, œuvres d'art exposées à l'extérieur du musée, savoir-faire traditionnels ou encore témoignages enregistrés sur vidéo sont autant d'exemples de cette tendance vers la dématérialisation, la mise en réseau des savoirs et des collections et les interactions multipolaires.

\section{Les enjeux actuels}

Malgré la tendance de la muséologie participative, le musée n'est pas systématiquement perçu comme un acteur social dans sa population et ses missions n'y aspirent pas forcément non plus. Cette tendance se manifeste par une accumulation d'actions ponctuelles et sectorielles pointant une communauté cible à un moment donné. Les actions participatives révèlent peut-être aussi une recherche de la part des musées de leur propre légitimité face à leur société, car ces initiatives participatives ont toujours leur origine du côté du musée et non des communautés. D'après Élise Dubuc, il reste à se poser une question : pourquoi
[8]

GERVEREAU, Laurent Vous avez dit musées? Tout savoir sur la crise culturelle. Paris : CNRS éditions, Carré sciences, 2006, p. 76-84.
[9]

Cette méthode a été développée par Hugues de Varine, expert en développement communautaire et ancien directeur de l'ICOM (1964-1974). 
cette "vogue" et ces discours actuellement ? Face au décalage important entre le discours de démocratisation et les impératifs de gestion, par ailleurs, Yves Bergeron postule que, "[d]epuis les années 1990, nous ne sommes pas dans une époque de démocratisation de la culture, au contraire." Et c'est sans doute en réponse à ce retour vers le musée élitiste que des projets participatifs sont développés.

Jean-François Leclerc souligne l'importance de l'engagement social et de la mise en place d'un réseau solide et durable. Une fois qu'un projet participatif est lancé avec une communauté, le musée doit assumer ou relayer adroitement cette relation, d'où l'importance de collaborer, avec des organismes communautaires par exemple. Ainsi, pour que la population considère le musée comme un acteur social important, celui-ci doit d'abord se positionner en tant que tel et s'ancrer profondément dans son environnement en s'engageant à long terme dans la communauté. Bref, comme le résume bien Élise Dubuc, «[l]e musée doit être dans le social pour être social.»
Finalement, il ressort du déroulement du colloque que les jeunes chercheurs se préoccupent fortement du rôle social du musée. Conséquemment, les organisatrices lancent un appel aux jeunes chercheurs en muséologie pour que cet événement se renouvelle chaque année! 